\title{
Eight-week consumption of milk enriched with omega 3 fatty acids raises their blood concentrations yet does not affect lipids and cardiovascular disease risk factors in adult healthy volunteers
}

\author{
Chiara Molinari ${ }^{a}$, Patrizia Risé ${ }^{\mathrm{b}}$, Claudia Guerra ${ }^{\mathrm{a}}$, Nicolò Mauro ${ }^{\mathrm{b}}$, Cecilia Piani $^{\mathrm{a}}$, \\ Emanuele Bosi ${ }^{\text {a,c }}$, Claudio Galli ${ }^{\mathrm{b}, *}$, Marina Scavini ${ }^{\mathrm{c}, * *}$ \\ a San Raffaele Vita-Salute University and San Raffaele Hospital E Scientific Institute, Milan, Italy \\ ${ }^{\mathrm{b}}$ Department of Pharmacological and Biomolecular Sciences, University of Milan, Italy \\ ${ }^{\mathrm{c}}$ Diabetes Research Institute, San Raffaele Hospital \& Scientific Institute, Milan, Italy
}

\section{A R T I C LE I N F O}

\section{Article history:}

Received 6 June 2014

Accepted 6 June 2014

\section{Keywords:}

Milk enriched with fish oil

Omega 3 fatty acids

Eicosapentaenoic acid

Docosahexaenoic acid

Thromboxane B2

Leukotriene B4

\begin{abstract}
A B S T R A C T
Adequate concentrations of omega 3 fatty acids (FA) may be maintained by consuming foods naturally rich in or functional foods enriched with those essential FA. We assessed the effects of an 8-week consumption of milk enriched with $400 \mathrm{mg}$ of omega 3 FA on blood lipid levels and selected cardiovascular risk factors in adult healthy volunteers. Participants $(n=157)$ were randomized to consume - for eight weeks - either $500 \mathrm{~mL} /$ day of $1 \%$ fat milk providing $150 \mathrm{mg}$ of eicosapentaenoic (EPA), $150 \mathrm{mg}$ of docosahexaenoic (DHA), and $100 \mathrm{mg}$ of $\alpha$-linolenic (ALA) acids or $500 \mathrm{~mL} /$ day of nonenriched $1 \%$ fat milk. At week 8 , we observed significant increments of blood EPA and DHA in the intervention group and a decrease in controls. No significant between-group differences at week 8 were observed in the lipid profile and other surrogate makers of cardiovascular disease, with the exception of homocysteine, whose levels remained stable in the intervention group, but increased in the control group $(p<0.001)$. In conclusion, daily consumption of $500 \mathrm{~mL}$ of $1 \%$ fat milk enriched with omega-3 FA for eight weeks leads to higher blood levels of EPA and DHA, confirming that milk is an optimal matrix for the provision of important micronutrients.
\end{abstract}

() 2014 Published by Elsevier B.V.

\section{Introduction}

Cardiovascular disease (CVD) is the leading cause of morbidity and mortality in Western countries [1] and its incidence and development can be modulated by diet. Among the various cardioprotective food components, omega 3 fatty acids have been the subject of extensive investigation. Even though supplemental omega 3 fatty acids do not, apparently, provide protection from cardiovascular disease [2], their consumption via diet, i.e. marine products are consistently associated with better cardiovascular prognosis [3]. Indeed, adequate circulating concentrations of longchain, namely eicosapentaenoic (EPA) and docosahexaenoic (DHA)

\footnotetext{
* Corresponding author at: Department of Pharmacological and Molecular Sciences, University of Milan, Via Balzaretti 9, 20233 Milan, Italy. Tel.: +39 0250318309.

** Corresponding author at: Diabetes Research Institute, San Raffaele Hospital \& Scientific Institute, Via Olgettina 60, 20132 Milan, Italy.

E-mail addresses: claudio.galli@unimi.it (C. Galli), scavini.marina@hsr.it (M. Scavini).
}

acids are directly associated with better cardiovascular health. Therefore, International scientific societies such as the ISSFAL (www.issfal.org) are suggesting the intake of $>250 \mathrm{mg} /$ day of EPA and DHA to maintain proper cardiovascular health. The mediumchain alpha-linolenic acid (ALA) is also essential and there is uncertainty regarding whether ALA has actions of its own in relation to the cardiovascular system and neural function $[4,5]$. Also, the rate of its elongation to EPA and DHA; in humans, appear to be minimal [6].

For the purpose of primary prevention of CVD in the general population, adequate circulating levels of omega 3 fatty acids may be maintained over a lifetime by consuming marine products or functional foods enriched with omega 3 fatty acids [7]. One suitable matrix for omega 3 provision is milk, because its fat is dispersed in micelles which efficiently increase the surface available for absorption of bioactive components in the gut $[8,9]$.

The aim of this study was to evaluate the effects of the shortterm administration of $500 \mathrm{~mL} /$ day of omega 3 fatty acidsenriched milk - providing $300 \mathrm{mg} /$ day of EPA and DHA - on blood fatty acid and lipid profile and on circulating cardiovascular risk 
factors [homocysteine, thromboxane $\mathrm{B}_{2}\left(\mathrm{TxB}_{2}\right)$ and leukotriene $\mathrm{B}_{4}$ $\left.\left(\mathrm{LTB}_{4}\right)\right]$ in adult healthy volunteers.

\section{Materials and methods}

\subsection{Study design}

We performed a double-blind, randomized, placebo-controlled trial conducted at the Scientific Institute San Raffaele (Milan, Italy). The protocol was approved by the Ethics Committee of the Scientific Institute San Raffaele and all participants gave written informed consent prior to enrolment. The study flow is shown in Fig. 1.

\subsection{Setting and participants}

The study was advertised through an e-mail sent to the entire Institution's database. Study participants were healthy adult volunteers. Eligibility criteria were: age 30-65 years, general good health, a body mass index (BMI) from 18.5 to 30. Exclusion criteria were: pregnancy or breast-feeding, intolerance to lactose or cow's milk proteins, dyslipidemia, dysthyroidism, smoking $\geq 10$ cigaarette/day, eating fish more than once a week, using medications affecting lipid levels (including drugs to treat dyslipidemia and heparin), intake of functional foods influencing lipid levels, and intake of omega 3 FA fatty acids as medications or food supplements during the six months preceding their enrolment in the study.

\subsection{Randomization and Interventions}

Allocation ratio to treatment groups was $1: 1$. We used the PEPI software to select random permuted blocks of size 20 [17]. Allocation information was sealed in sequentially numbered opaque envelopes prepared by a biostatistician not involved in the trial. Participants in the intervention group were asked to drink - during eight weeks - $500 \mathrm{~mL} /$ day of $1 \%$ fat milk enriched with fish oil, containing $400 \mathrm{mg}$ of omega 3 fatty acids ( $150 \mathrm{mg}$ each of EPA and DHA and $100 \mathrm{mg}$ of alpha linolenic acid); vitamins C, E, B6, and B12; and folic acid. Participants in the control group were asked to drink - during eight weeks $-500 \mathrm{~mL} /$ day of $1 \%$ fat milk not enriched with fish oil. The two types of milk used in this study were packed in white $500 \mathrm{~mL}$ Tetrapack ${ }^{\mathrm{TM}}$ containers labelled either "milk A" or "milk B" by Parmalat (Collecchio, PR, Italy). The composition of the two types of milk used in this study is reported in Table 1.

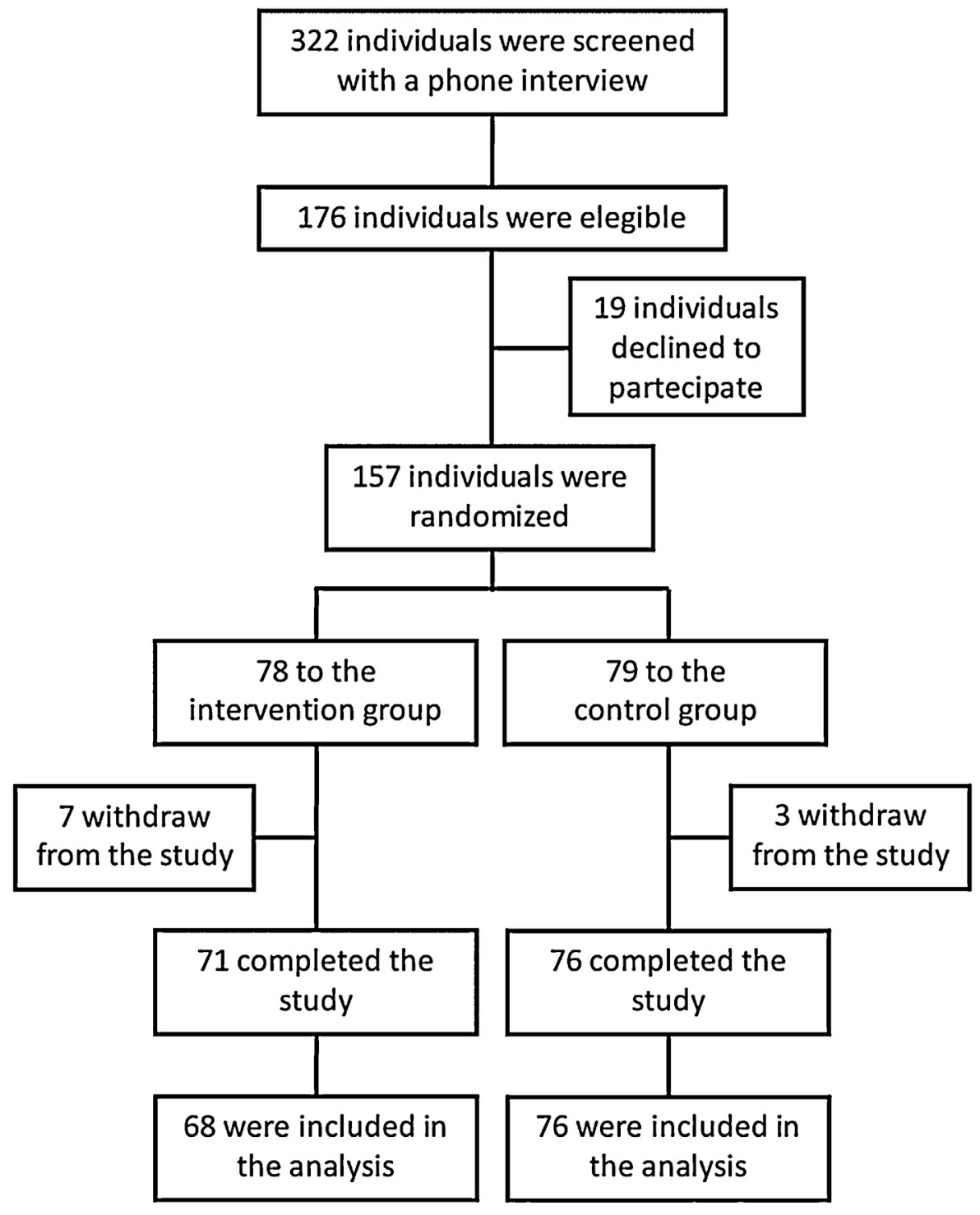

Fig. 1. Study flow. 
Table 1

Nutritional values (per $100 \mathrm{~mL}$ ) of the two types of milk used in this study, with percentage of Recommended Dietary Allowance in parentheses.

\begin{tabular}{lll}
\hline & Supplemented milk & Control milk \\
\hline Energy (kcal) & 43 & 41 \\
Energy (kJ) & 182 & 175 \\
Proteins (g) & 3.2 & 3.2 \\
Carbohydrates (g) & 4.9 & 4.9 \\
- sugars & 4.9 & 4.9 \\
Fats (g) & 1.2 & 1 \\
- saturated (g) & 0.7 & - \\
- mono-saturated (g) & 0.36 & - \\
- poly-unsaturated (g) & 0.14 & - \\
- omega 3 fatty acids (mg) & 80 & - \\
- DHA+EPA (mg) & 60 & - \\
- alpha-linolenic acid & 20 & - \\
$\quad$ and others (mg) & & 0.05 \\
Sodium (g) & 0.05 & $120(15 \%)$ \\
Calcium (mg) & $120(15 \%)$ & - \\
Vitamin C (mg) & $24(40 \%)$ & - \\
Vitamin E (mg) & $4(40 \%)$ & - \\
Vitamin B6 (mg) & $0.8(40 \%)$ & - \\
Vitamin B12 ( $\mu$ g) & $0.4(40 \%)$ & - \\
Folic acid ( $\mu$ g) & $80(40 \%)$ & \\
\hline
\end{tabular}

\subsection{Follow-up and outcomes}

For the 8 weeks of the study, participants were asked not to change their usual diet, to eat fish only once a week, to limit the intake of other foods rich in omega 3 fatty acids, i.e., shellfish, nuts, rapeseed oil, and to avoid food supplements containing such fatty acids. Participants were asked to record - in a diary - the amount of milk consumed daily and the fish servings eaten during the study.

At baseline, 4, and 8 weeks, venous blood samples were drawn from all participants after an overnight fast of at least $10 \mathrm{~h}$. Fatty acid profile, white blood cells (WBC), fibrinogen (FG), lipid profile (total and HDL cholesterol, tryglycerides), homocysteine, leukotriene $\mathrm{B}_{4}\left(\mathrm{LTB}_{4}\right)$ and thromboxane $\mathrm{B}_{2}\left(\mathrm{TxB}_{2}\right)$ levels in serum produced under controlled conditions were assessed (see below). At the end of the study, a questionnaire was administered to collect data about body weight, side effects, and palatability of the type of milk used in the study.

\subsection{Laboratory assays}

The analytical method used for measuring blood fatty acid profiles, developed as previously described [10], was based on blood collection on a special adsorbent (Sigma-Aldrich, St. Louis, MO) embedded with the antioxidant butyl hydroxyl toluene (BHT) in order to prevent the degradation of polyunsaturated fatty acids. Samples were stored at $4{ }^{\circ} \mathrm{C}$ in individual airtight sealed cellophane envelopes. The whole blood fatty acid profile was then assessed by gas chromatography, following a procedure based on the preparation of fatty acid methyl esters (FAMEs) by direct trans esterification of the sample, without lipid extraction [10].

Blood aliquots were allowed to clot at $37{ }^{\circ} \mathrm{C}$ for $1 \mathrm{~h}$. Serum was then separated by centrifugation at $2100 \times g$ for $20 \mathrm{~min}$ at $4{ }^{\circ} \mathrm{C}$, aliquoted, and stored in a freezer. Concentrations of $\mathrm{TXB}_{2}$ were measured in this serum. Other blood aliquots were immediately added with the calcium-ionophore A23187 (50 mM; Calbiochem, Darmstadt, Germany) and incubated for $30 \mathrm{~min}$ at $37{ }^{\circ} \mathrm{C}$ to stimulate $\mathrm{LTB}_{4}$ production. At the end of the incubation period, samples were centrifuged to separate serum. The serum concentrations of $\mathrm{LTB}_{4}$ and $\mathrm{TXB}_{2}$ were measured by immunoassay (Cayman Chemical, Ann Arbor, MI) [11] on a subset of randomly selected samples and were expressed as percentage of the concentrations at week 0 .
White blood cells were counted using an automated counter (Coulter STKS or Coulter LH750, Beckman Coulter, Brea, CA). Fibrinogen was measured using the coagulation method (STA - R, Diagnostica Stago, Parsippany, NJ). Lipid profile was measured by an enzymatic-colorimetric method (Advia 2400, Siemens Healthcare Diagnostics, Deerfield, IL). LDL-cholesterol was calculated using the Friedewald formula for triglyceride levels $<400 \mathrm{mg} / \mathrm{dL}$ [12]. Homocysteine was measured by an enzymatic method with the Omocysteine IMX System Assay (Abbott Diagnostic Division, Rome, Italy). Sensibility was $<0.50 \mu \mathrm{mol} / \mathrm{L}$, intra-assay CV was $2.2 \%$, while inter-assay was $5.2 \%$.

\subsection{Statistical analysis}

All participants who had their blood drawn at 0,4 , and 8 weeks were included in the analysis. When reporting the general characteristics of participants, categorical variables are presented as frequency and proportion, continuous variables as median and interquartile range (IQR). Longitudinal variables were analyzed using mixed linear model [13] with randomized group, visit and randomized group-by-visit interaction as fixed effects. An unstructured variance-covariance matrix was used to model the correlation between repeated measurements within each participant. Restricted maximum likelihood estimates and two-sided 95\% confidence intervals $(\mathrm{CI})$ of the mean difference between randomized groups at each study week were calculated using the Newton-Raphson algorithm. Changes of $\mathrm{LTB}_{4}$ and $\mathrm{TxB}_{2}$ at week 4 and 8 , expressed as percent of week 0 , were compared using the Student's $t$ test for unpaired data. A $p$ value of $<0.05$ was considered statistically significant. Statistical analysis was performed using the statistical software Stata, version 11.2 (Stata Corporation, College Station, TX).

\section{Results}

Participants in the two treatment groups were similar for age [median age: 41 years (IQR 36-50) intervention group; 43 years (38-51) control group] and sex (57 and 55\% females in the intervention group and control group, respectively). Compliance with milk and fish consumption during the study was good: 60 and $63 \%$ in the intervention group and in the control group, respectively, consumed $500 \mathrm{~mL} /$ day of milk as required by the study protocol. The median cumulative amount of milk not consumed was similar in the two groups [1200 mL (IQR 600$2350 \mathrm{~mL})$ in the intervention group and $900 \mathrm{~mL}(500-2450 \mathrm{~mL})$ in the control group, $p=0.785]$. The number of fish servings consumed during the study was also similar in the two groups [6 (IQR 2-8) in the intervention group and $6(4-8)$ in the control group, $p=0.070]$.

Both milks used in the study were judged palatable by the majority of study participants (70 and $71 \%$ in the intervention group and control group, respectively, $p=0.924$ ). Fish flavor in the milks used in the study was reported by only three participants in the intervention group and one participant in the control group, $p=0.356$. Side effects were all mild, occurred at a similar rate in the two groups, and were possibly associated to the volume of milk consumed daily, rather than to the presence of omega 3 fatty acids (Table 2).

Blood concentrations of omega 3 fatty acids during the study are shown in Fig. 2 and Supplementary Table 1. At baseline, blood concentrations of omega 3 fatty acids were similar in the two groups. During the study, ALA hematic concentrations decreased, although not significantly, in both groups. Docosapentaenoic acid (DPA) decreased in both groups, but the decrease from baseline was significant only in the control group, with a non-significant between-group difference at week 4 and 8. EPA hematic 
Table 2

Side effects reported during the study.

\begin{tabular}{cccc}
\hline & $\begin{array}{c}\text { Intervention } \\
\text { group } N=68\end{array}$ & $\begin{array}{l}\text { Control } \\
\text { group } N=76\end{array}$ & $p$ Value \\
\hline Gastrointestinal $^{\mathrm{a}}$ & $22(31)$ & $26(35)$ & 0.636 \\
Abdominal pain & $5(7)$ & $6(8)$ & 0.827 \\
Meteorism & $11(15)$ & $15(20)$ & 0.477 \\
$\begin{array}{l}\text { Increased rate } \\
\text { of defecation }\end{array}$ & $8(11)$ & $12(16)$ & 0.406 \\
Soft faeces & $11(15)$ & $13(17)$ & 0.764 \\
Constipation & 0 & $2(2.7)$ & 0.497 \\
Cutaneous rash & $3(4)$ & 0 & 0.245 \\
\hline
\end{tabular}

Data are presented as number of patients with a specific side effect and percent in parentheses.

a Patients may have reported more than one side-effect.

concentrations increased (+28\%) significantly in the intervention group, but they decreased significantly $(-10 \%)$ in the control group, with a significant between-group difference at weeks 4 and 8. DHA increased $(+7 \%)$ in the intervention group, although not significantly, and decreased significantly $(-8 \%)$ in the control group, with a significant between-group difference at weeks 4 and 8. During the study, the changes in ALA, EPA, and DHA were similar between males and females, while DPA levels were lower at baseline and decreased more during the study among females than males (Supplementary Table 2).

Supplementary tables related to this article can be found, in the online version, at doi:10.1016/j.phanu.2014.06.001.

The levels of non-omega 3 fatty acids during the study are shown in Table 3. We observed no significant between-group differences in the levels of non-omega 3 fatty acids with two exceptions: (1) gadolenic acid [20:1] was higher at baseline in the control group than in the intervention group. During the study gadolenic acid increased slightly in the control group and remained stable in the intervention group, with a significant, although small, between-group difference at week 8 . At baseline; (2) the omega 6 dihomo-gamma-linolenic acid [20:3 n-6] was significantly higher in the intervention group than in controls, but decreased during the 8 weeks of study, reaching levels similar to controls.

Blood levels of total saturated (SFA), monounsaturated (MUFA), omega 3, omega 6, and polyunsaturated (PUFA) fatty acids during the study are shown in Table 4. At baseline, hematic levels of SFA, MUFA, omega 3, omega 6 and PUFA were similar in the two groups. Total SFA levels increased modestly yet significantly and with a similar trend in both groups, without significant between-group differences at weeks 4 and 8 . There was a tendency to an increase in MUFA levels in both groups, although this increase was significant only in the control group, without significant between-group differences at weeks 4 and 8 . Total PUFAs decreased significantly and similarly in both groups, without significant between-group differences at weeks 4 and 8 . Total omega 3 fatty acid levels increased, although not significantly, in the intervention group and decreased significantly in the control group, with significant between-group differences at weeks 4 and 8 . Total omega 6 levels decreased significantly and with a similar trend in both groups, without significant betweengroup differences at weeks 4 and 8 .

Total cholesterol, HDL-cholesterol, LDL-cholesterol, tryglycerides, fibrinogen and white blood cell counts were similar in the two groups at baseline and did not change throughout the study, as shown in Table 5. Homocysteine levels increased significantly in the control group and remained unchanged in the intervention group, with a significant between-group difference at week 8 $(p<0.0001)$. During the study, there were no significant changes in $\mathrm{TxB}_{2}$ or $\mathrm{LTB}_{4}$ serum levels, as shown in Fig. 3.

\section{Discussion}

Our study shows that the daily consumption of $500 \mathrm{~mL}$ of milk enriched with $150 \mathrm{mg}$ each of EPA and DHA and $100 \mathrm{mg}$ of ALA significantly increases their hematic concentrations, especially those of EPA and, slightly, those of DHA. These increases are associated with a decrease of ALA and DPA, confirming that milk is an efficient vehicle for the absorption of EPA and DHA from the gut.
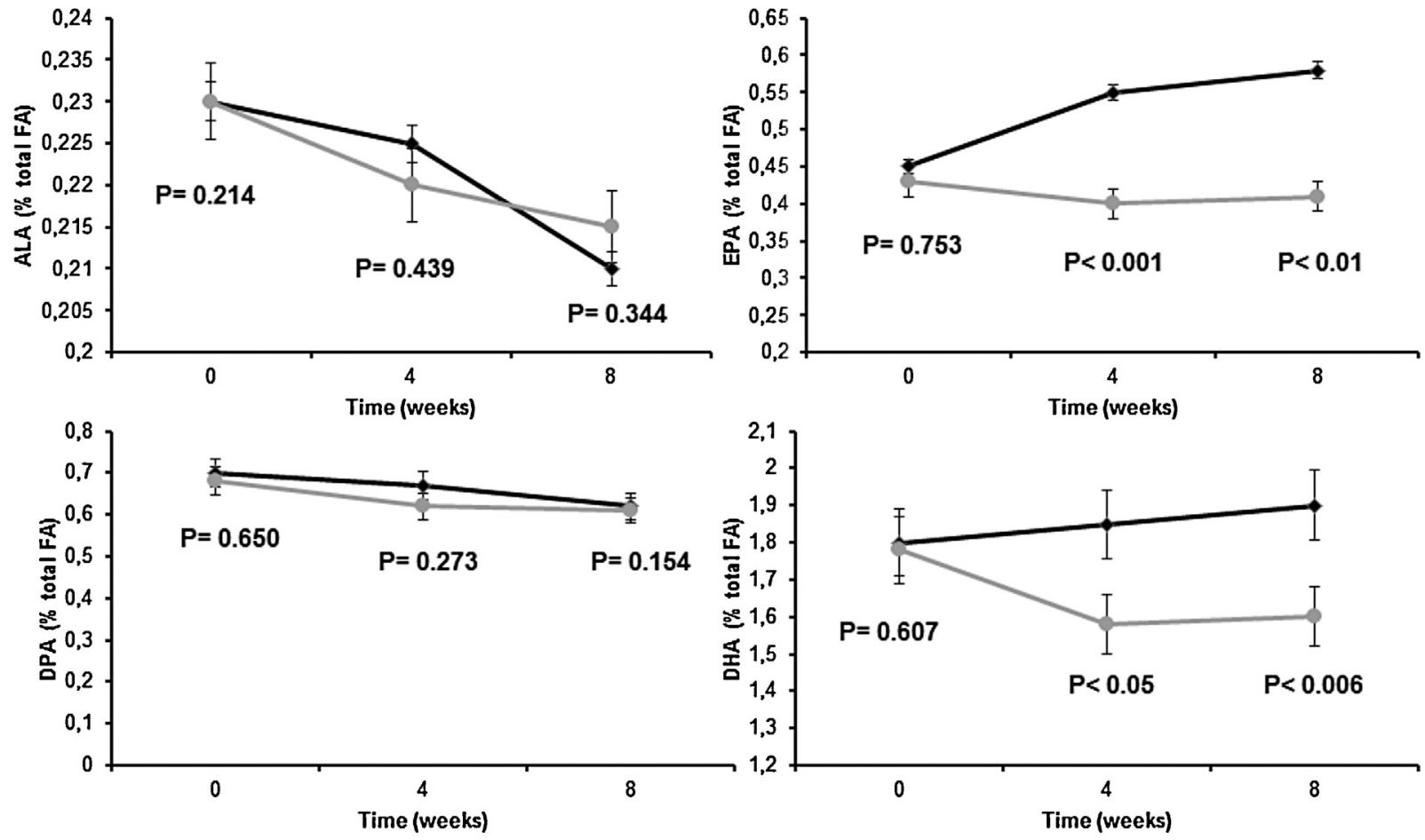

Fig. 2. Mean blood levels ( \pm standard deviation) of omega 3 fatty acids during the study in the intervention (black symbols) and control (white symbols) groups. 
Table 3

Blood levels of non-omega 3 fatty acids during the study in the intervention and control groups.

\begin{tabular}{|c|c|c|c|c|c|c|}
\hline & Week & $\begin{array}{l}\text { Intervention } \\
\text { group } N=68\end{array}$ & $\begin{array}{l}\text { Direction of change } \\
\text { overtime } p \text { value, } \\
\text { week } 8 \text { vs. week } 0\end{array}$ & $\begin{array}{l}\text { Control group } \\
N=76\end{array}$ & $\begin{array}{l}\text { Direction of change } \\
\text { overtime } p \text { value, } \\
\text { week } 8 \text { vs week } 0\end{array}$ & $p$ Value \\
\hline \multirow[t]{3}{*}{$16: 0$ (\% of total FA) } & 0 & 25.632 (1.799) & $\uparrow$ & $25.612(1.761)$ & $\uparrow$ & 0.951 \\
\hline & 4 & $26.370(2.217)$ & 0.0002 & $26.168(2.000)$ & 0.0117 & 0.547 \\
\hline & 8 & $26.539(2.148)$ & & $26.186(2.077)$ & & 0.290 \\
\hline \multirow[t]{3}{*}{$18: 0$ (\% of total FA) } & 0 & $11.346(1.020)$ & $\uparrow$ & $11.221(1.008)$ & $\downarrow$ & 0.476 \\
\hline & 4 & $11.527(1.032)$ & 0.273 & $11.389(1.064)$ & 0.103 & 0.435 \\
\hline & 8 & $11.428(1.159)$ & & $11.185(1.060)$ & & 0.169 \\
\hline \multirow[t]{3}{*}{$20: 0$ (\% of total FA) } & 0 & $0.438(0.081)$ & $\downarrow$ & $0.432(0.064)$ & $\downarrow$ & 0.617 \\
\hline & 4 & $0.421(0.075)$ & 0.0051 & $0.423(0.070)$ & 0.1130 & 0.877 \\
\hline & 8 & $0.417(0.087)$ & & $0.419(0.070)$ & & 0.873 \\
\hline \multirow[t]{3}{*}{$22: 0$ (\% of total FA) } & 0 & $1.499(0.198)$ & $\downarrow$ & $1.456(0.210)$ & $\downarrow$ & 0.258 \\
\hline & 4 & $1.444(0.248)$ & 0.172 & $1.409(0.231)$ & 0.111 & 0.357 \\
\hline & 8 & $1.449(0.242)$ & & $1.411(0.223)$ & & 0.311 \\
\hline \multirow[t]{3}{*}{$24: 0$ (\% of total FA) } & 0 & $2.536(0.374)$ & $\uparrow$ & $2.515(0.383)$ & $\downarrow$ & 0.740 \\
\hline & 4 & $2.570(0.416)$ & 0.704 & $2.550(0.364)$ & 0.158 & 0.762 \\
\hline & 8 & $2.559(0.411)$ & & $2.473(0.412)$ & & 0.194 \\
\hline \multirow[t]{3}{*}{$16: 1$ (\% of total FA) } & 0 & $1.620(0.621)$ & $\uparrow$ & $1.636(0.598)$ & $\uparrow$ & 0.880 \\
\hline & 4 & $1.676(0.628)$ & 0.168 & $1.761(0.657)$ & 0.0427 & 0.423 \\
\hline & 8 & $1.718(0.637)$ & & $1.770(0.662)$ & & 0.622 \\
\hline \multirow[t]{3}{*}{$18: 1$ (\% of total FA) } & 0 & $18.962(2.085)$ & $\uparrow$ & $19.014(2.470)$ & $\uparrow$ & 0.903 \\
\hline & 4 & $19.220(2.420)$ & 0.275 & $19.620(2.522)$ & 0.0056 & 0.346 \\
\hline & 8 & $19.373(2.576)$ & & $19.815(3.047)$ & & 0.298 \\
\hline \multirow[t]{3}{*}{$18: 1$ n 7 (\% of total FA) } & 0 & $1.671(0.472)$ & $\uparrow$ & $1.653(0.401)$ & $\uparrow$ & 0.803 \\
\hline & 4 & $1.681(0.424)$ & 0.581 & $1.724(0.453)$ & 0.061 & 0.561 \\
\hline & 8 & $1.709(0.502)$ & & $1.681(0.368)$ & & 0.699 \\
\hline \multirow[t]{3}{*}{$20: 1$ (\% of total FA) } & 0 & $0.200(0.040)$ & $=$ & $0.213(0.049)$ & $\uparrow$ & 0.063 \\
\hline & 4 & $0.196(0.032)$ & 0.473 & $0.217(0.040)$ & 0.619 & 0.002 \\
\hline & 8 & $0.200(0.038)$ & & $0.218(0.040)$ & & 0.010 \\
\hline \multirow[t]{3}{*}{$22: 1$ (\% of total FA) } & 0 & $0.325(0.549)$ & $\downarrow$ & $0.454(0.628)$ & $\downarrow$ & 0.063 \\
\hline & 4 & $0.159(0.239)$ & 0.0002 & $0.201(0.302)$ & $<0.0001$ & 0.545 \\
\hline & 8 & $0.094(0.051)$ & & $0.134(0.419)$ & & 0.564 \\
\hline \multirow[t]{3}{*}{$24: 1$ (\% of total FA) } & 0 & $2.832(0.490)$ & $\uparrow$ & $2.910(0.570)$ & $=$ & 0.383 \\
\hline & 4 & $2.934(0.487)$ & 0.116 & $3.001(0.544)$ & 0.192 & 0.455 \\
\hline & 8 & $2.970(0.566)$ & & $2.921(0.532)$ & & 0.583 \\
\hline \multirow[t]{3}{*}{$20: 3$ n 9 (\% of total FA) } & 0 & $0.125(0.051)$ & $\downarrow$ & $0.122(0.053)$ & $\uparrow$ & 0.776 \\
\hline & 4 & $0.120(0.049)$ & 0.225 & $0.120(0.054)$ & 0.204 & 0.333 \\
\hline & 8 & $0.118(0.048)$ & & $0.130(0.051)$ & & 0.158 \\
\hline \multirow[t]{3}{*}{$18: 2$ n 6 (\% of total FA) } & 0 & $18.997(2.388)$ & $\downarrow$ & $19.252(2.541)$ & $\downarrow$ & 0.500 \\
\hline & 4 & $18.610(2.450)$ & 0.109 & $18.806(2.574)$ & 0.106 & 0.642 \\
\hline & 8 & $18.399(2.503)$ & & $18.815(2.663)$ & & 0.324 \\
\hline \multirow[t]{3}{*}{$18: 3$ n 6 (\% of total FA) } & 0 & $0.260(0.095)$ & $\downarrow$ & $0.268(0.134)$ & $\downarrow$ & 0.688 \\
\hline & 4 & $0.266(0.126)$ & 0.746 & $0.261(0.120)$ & 0.890 & 0.817 \\
\hline & 8 & $0.257(0.117)$ & & $0.264(0.115)$ & & 0.709 \\
\hline \multirow[t]{3}{*}{$20: 3$ n 6 (\% of total FA) } & 0 & $1.448(0.325)$ & $\downarrow$ & $1.319(0.269)$ & $\uparrow$ & 0.031 \\
\hline & 4 & $1.390(0.310)$ & 0.0124 & $1.365(0.299)$ & 0.1148 & 0.671 \\
\hline & 8 & $1.365(0.316)$ & & $1.427(0.545)$ & & 0.300 \\
\hline \multirow[t]{3}{*}{$20: 4$ n 6 (\% of total FA) } & 0 & 7.745 (1.387) & $\downarrow$ & 7.587 (1.519) & $\downarrow$ & 0.530 \\
\hline & 4 & $7.063(1.420)$ & 0.0001 & $7.093(1.487)$ & 0.0107 & 0.906 \\
\hline & 8 & $6.993(1.474)$ & & $7.224(1.739)$ & & 0.359 \\
\hline \multirow[t]{3}{*}{$22: 4$ n 6 (\% of total FA) } & 0 & $0.934(0.250)$ & $\downarrow$ & $0.908(0.278)$ & $\downarrow$ & 0.568 \\
\hline & 4 & $0.829(0.245)$ & 0.0001 & $0.870(0.291)$ & 0.055 & 0.357 \\
\hline & 8 & $0.810(0.284)$ & & $0.841(0.254)$ & & 0.488 \\
\hline \multirow[t]{3}{*}{$22: 5$ n 6 (\% of total FA) } & 0 & $0.253(0.100)$ & $\downarrow$ & $0.263(0.141)$ & $\downarrow$ & 0.539 \\
\hline & 4 & $0.221(0.101)$ & 0.0026 & $0.231(0.109)$ & 0.0220 & 0.552 \\
\hline & 8 & $0.215(0.093)$ & & $0.225(0.078)$ & & 0.557 \\
\hline
\end{tabular}

Data are means with standard deviations in parentheses. FA, fatty acids.

Administration of omega 3-enriched milk did not modify any of the surrogate markers of cardiovascular disease we assessed.

The significant decrease in circulating levels of omega 3 observed in the control group was likely due to the protocol requirement of maintaining a low consumption of foods naturally rich in such fatty acids. In the intervention group the supplementation of EPA and DHA in the milk not only efficiently compensated for the decreased dietary intake of omega 3 with food, but resulted in a significant increase in circulating levels of EPA (but not DHA). On the other side, the lack of a significant increment of blood total 
Table 4

Blood levels of saturated, monounsaturated, omega 3, omega 6, and polyunsaturated fatty acids during the study in the intervention and control groups.

\begin{tabular}{|c|c|c|c|c|c|c|}
\hline & Week & $\begin{array}{l}\text { Intervention } \\
\text { group } N=68\end{array}$ & $\begin{array}{l}\text { Direction of change } \\
\text { overtime } p \text { value, } \\
\text { week } 8 \text { vs week } 0\end{array}$ & $\begin{array}{l}\text { Control group } \\
N=76\end{array}$ & $\begin{array}{l}\text { Direction of change } \\
\text { overtime } p \text { value, } \\
\text { week } 8 \text { vs week } 0\end{array}$ & $\begin{array}{l}p \text { Value, intervention } \\
\text { vs control group }\end{array}$ \\
\hline \multirow[t]{3}{*}{ SFA (\% of total FA) } & 0 & $41.367(2.374)$ & $\uparrow$ & $41.149(2.234)$ & $\uparrow$ & 0.609 \\
\hline & 4 & $42.331(2.670)$ & 0.0054 & $41.939(2.398)$ & 0.0485 & 0.357 \\
\hline & 8 & $42.365(2.925)$ & & $41.653(2.690)$ & & 0.095 \\
\hline \multirow[t]{3}{*}{ MUFA (\% of total FA) } & 0 & $25.612(2.436)$ & $\uparrow$ & $25.881(2.680)$ & $\uparrow$ & 0.569 \\
\hline & 4 & $25.867(2.618)$ & 0.323 & $26.524(2.956)$ & 0.0351 & 0.164 \\
\hline & 8 & $26.064(2.880)$ & & $26.539(3.273)$ & & 0.314 \\
\hline \multirow[t]{3}{*}{ Omega $3 \mathrm{FA}$ (\% of total FA) } & 0 & $3.174(1.379)$ & $\uparrow$ & 3.199 (1.227) & $\downarrow$ & 0.907 \\
\hline & 4 & $3.302(1.202)$ & 0.199 & $2.876(1.300)$ & 0.0016 & 0.045 \\
\hline & 8 & $3.386(1.148)$ & & $2.943(1.351)$ & & 0.037 \\
\hline \multirow[t]{3}{*}{ Omega $6 \mathrm{FA}$ (\% of total FA) } & 0 & $29.637(2.806)$ & $\downarrow$ & $29.308(4.448)$ & $\downarrow$ & 0.621 \\
\hline & 4 & $28.379(3.242)$ & 0.0007 & $28.311(4.541)$ & 0.0155 & 0.918 \\
\hline & 8 & 28.039 (3.519) & & $28.453(4.769)$ & & 0.535 \\
\hline Total PUFA & 0 & $32.871(3.281)$ & $\downarrow$ & $32.855(3.710)$ & $\downarrow$ & 0.981 \\
\hline \multirow[t]{2}{*}{ (\% of total FA) } & 4 & $31.535(3.914)$ & 0.0069 & $31.276(4.055)$ & 0.0008 & 0.694 \\
\hline & 8 & $31.349(4.260)$ & & $31.565(4.400)$ & & 0.744 \\
\hline
\end{tabular}

Data are means with standard deviation in parentheses. SFA, saturated fatty acids; MUFA, monounsaturated fatty acids, FA, fatty acids.

omega 3 fatty acids in the intervention group is related to the reduction of ALA and DPA. This may hypothetically due to reduction of the endogenous conversion of the precursor ALA to its longer-chain products (EPA and DHA), through desaturation and elongation processes, as a consequence of the intake of the preformed compounds. However, other factors cannot be excluded.

In terms of circulating concentrations, we also observed differences between males and females. Lower baseline levels of DPA among females have been previously reported [14] and are likely due to a more efficient conversion of DPA to DHA among females than males: this mechanism is likely to apply also to the lower levels of DPA observed throughout the study.

Because the milk we administered provided $300 \mathrm{mg} /$ day of long-chain omega 3 PUFA, increments of blood concentrations of these fatty acids were of course lower than those measured in individuals receiving supplementation with high doses, i.e., more than $1.5 \mathrm{~g} /$ day daily of the same fatty acids. Unlike other studies testing milk supplemented with low doses, i.e., less than $400 \mathrm{mg} / \mathrm{of}$ omega 3 in healthy volunteers $[8,15]$, patients with hypertriglyceridemia [16], metabolic syndrome [17], or increased cardiovascular risk [18], we did not observe any effect on the lipid profile. Many factors may explain these discrepant findings. First, the studies published thus far in healthy volunteers $[8,15]$ and in patients with hypertriglyceridemia [16] recruited small, i.e. $\leq 30$ numbers of subjects and did not include control groups; therefore, it is difficult to attribute the observed changes in the lipid profile to the omega 3 supplementation. Second, when enrolled in a randomized clinical trial, patients with metabolic syndrome [17] or increased cardiovascular risk [18] may have had a greater

Table 5

Total, HDL, and LDL cholesterol, triglycerides, fibrinogen, white blood cell counts, and homocysteine levels during the study in the intervention and control groups.

\begin{tabular}{|c|c|c|c|c|c|c|}
\hline & Week & $\begin{array}{l}\text { Intervention } \\
\text { group } N=68\end{array}$ & $\begin{array}{l}\text { Direction of change } \\
\text { overtime } p \text { value, } \\
\text { week } 8 \text { vs week } 0\end{array}$ & $\begin{array}{l}\text { Control group } \\
N=76\end{array}$ & $\begin{array}{l}\text { Direction of change } \\
\text { overtime } p \text { value, } \\
\text { week } 8 \text { vs week } 0\end{array}$ & $p$ Value \\
\hline \multirow[t]{3}{*}{ Total cholesterol (mg/dL) } & 0 & $210(35.7)$ & $\downarrow$ & $201(34.8)$ & $\downarrow$ & 0.116 \\
\hline & 4 & $203(36.2)$ & 0.0016 & $196(34.0)$ & 0.0932 & 0.241 \\
\hline & 8 & $202(34.9)$ & & $196(32.0)$ & & 0.288 \\
\hline \multirow[t]{3}{*}{ LDL cholesterol (mg/dL) } & 0 & $134(31.3)$ & $\downarrow$ & $128(30.0)$ & $\downarrow$ & 0.235 \\
\hline & 4 & $128(28.1)$ & 0.0034 & $124(28.0)$ & 0.078 & 0.362 \\
\hline & 8 & $127(29.2)$ & & $124(28.1)$ & & 0.482 \\
\hline \multirow[t]{3}{*}{ HDL cholesterol (mg/dL) } & 0 & $57(16.5)$ & $\downarrow$ & $54(12.5)$ & $\downarrow$ & 0.343 \\
\hline & 4 & $53(15.9)$ & $<0.0001$ & $51(13.1)$ & 0.0003 & 0.579 \\
\hline & 8 & $53(15.0)$ & & $52(12.1)$ & & 0.792 \\
\hline \multirow[t]{3}{*}{ Triglycerides (mg/dL) } & 0 & $93(44.1)$ & $\uparrow$ & $91(39.8)$ & $\uparrow$ & 0.916 \\
\hline & 4 & $106(71.9)$ & 0.173 & $101(52.9)$ & 0.125 & 0.616 \\
\hline & 8 & $108(70.7)$ & & $100(55.5)$ & & 0.702 \\
\hline \multirow{3}{*}{$\begin{array}{l}\text { White blood cell counts } \\
\qquad\left(\times 1000 / \mathrm{mm}^{3}\right)\end{array}$} & 0 & $6.24(1.70)$ & $\downarrow$ & $5.74(1.15)$ & $\downarrow$ & 0.032 \\
\hline & 4 & $6.04(1.42)$ & 0.483 & $5.82(1.03)$ & 0.780 & 0.326 \\
\hline & 8 & $6.14(1.60)$ & & $5.81(1.40)$ & & 0.155 \\
\hline \multirow[t]{3}{*}{ Fibrinogen $(\mathrm{mg} / \mathrm{dL})$} & 0 & $295(57.8)$ & $\uparrow$ & $299(61.8)$ & $\uparrow$ & 0.682 \\
\hline & 4 & $313(51.5)$ & 0.0041 & $313(62.4)$ & 0.0002 & 0.978 \\
\hline & 8 & $316(79.3)$ & & $320(63.2)$ & & 0.655 \\
\hline \multirow[t]{2}{*}{ Homocysteine ( $\mu \mathrm{mol} / \mathrm{L})$} & 0 & $10.9(3.8)$ & $\downarrow$ & $10.9(2.8)$ & $\uparrow$ & 0.157 \\
\hline & 8 & $10.0(3.6)$ & 0.939 & $12.6(3.9)$ & $<0.0001$ & 0.004 \\
\hline
\end{tabular}

Data are means with standard deviations in parentheses. LDL, low-density lipoprotein; HDL; high-density lipoprotein 

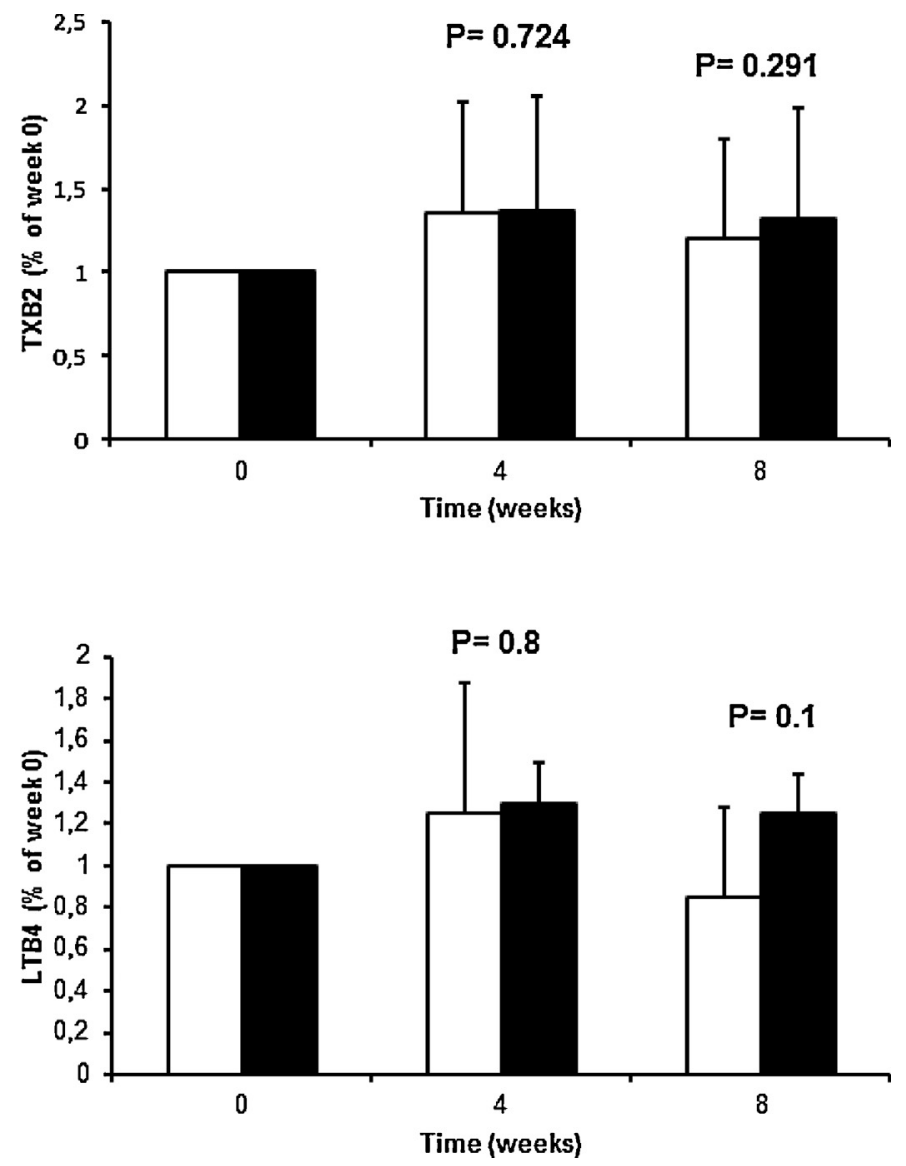

Fig. 3. Mean change (SD) of serum thromboxane $B_{2}$ (upper panel) and leukotriene $B_{4}$ (lower panel) during the study, expressed as percentage of levels at week 0 , in the intervention (black columns) and control (white columns) groups.

motivation to comply with omega 3 supplementation than the healthy volunteers of the current study, although neither of the two trials reported on the compliance of participants or side effects. In addition, effects on lipid and metabolic parameters are more likely to be observed in patients with alterations in these parameters. Furthermore, in the trial by Fonolla et al. [18] the intervention lasted significantly longer than in our trial (respectively 8 vs 12 [17] and 52 [18] weeks) and this may explain, at least in part, the fact that we did not observe any effect of omega 3 supplementation on the lipid profile. Last, but not least, while the increments in levels of circulating EPA and DHA in the 52 weeks intervention [18] were rather low and somehow comparable to those in our study, the longer duration of the treatment may explain the favorable effects on risk parameters obtained.

In the other studies with supplements of omega 3-rich milk, possibly as the result of complex factors, increments of these fatty acids were quite higher than in our study, e.g. 90 and 20\% increments of plasma EPA and DHA in the study by Carrero et al. [16] vs. 27 and 7\%, respectively in our investigation.

During our study we observed an increase in circulating homocysteine levels in the control group, but not in the intervention group where homocysteine levels did not change overtime, likely because of the addition of folic acid, vitamin B6 and B12, i.e. compounds that decrease homocysteine concentrations [19], to the milk supplemented with EPA and DHA and $\alpha-$ linolenic acid.

Our study has several limitations. First, the limited duration of the intervention: milk enriched with omega 3 fatty acids is a functional food meant to be consumed for longer periods of time than eight weeks if the objective is disease prevention, especially in the cardiovascular area. Second, we administered a low dose of omega 3: when milk is used as a vehicle for EPA and DHA supplementation, increasing the dose would greatly decrease palatability and, therefore, compliance. Third, participants were asked to limit the intake of foods naturally rich in omega 3 fatty acids during the study: we do not know whether longer supplementation, higher daily doses of EPA and DHA, or no limitations in the intake of foods rich in omega 3 may have produced different results in terms of lipid profile and CVD markers.

Our study has also several strengths. First, we enrolled a large number of participants from a working population who are likely to represent the consumers of functional foods with the purpose of CVD prevention. Second, as part of the study we collected data on compliance, palatability, and side effects on all participants, providing information on the feasibility of this approach to supplement omega 3 to a large population. Third, at difference with the design in some of the other cited studies $[8,15,16]$, we included a control group consuming a non-enriched milk was included.

In conclusion, daily consumption of $500 \mathrm{~mL}$ of milk supplemented with medium- and long-chain omega 3 PUFA (600 mg/L) increases plasma concentrations of EPA and DHA; this regimen does not affect total, HDL, and LDL cholesterol; triglycerides; white blood cell counts; fibrinogen; $\mathrm{LTB}_{4}$ and $\mathrm{TxB}_{2}$. Functional milk is a feasible approach to supplement omega 3 to the general population and maintain their appropriate circulating concentrations.

\section{Layperson's summary}

Adequate concentrations of omega 3 fatty acids may be maintained by consuming foods naturally rich in or functional foods enriched with those essential fatty acids. We assessed the effects of an 8-week consumption of milk enriched with $400 \mathrm{mg}$ of omega 3 fatty acids on blood fatty acid levels and lipids and selected cardiovascular risk factors. At week 8,we observed significant increments of blood EPA and DHA in the intervention group and a decrease of these fatty acids in controls. No significant between-group differences at week 8 were observed in the lipid profile and other surrogate makers of cardiovascular disease, with the exception of homocysteine, whose levels remained stable in the intervention group, but increased in the control group. In conclusion, daily consumption of $500 \mathrm{~mL}$ of $1 \%$ fat milk enriched with omega-3 fatty acids for eight weeks leads to higher blood levels of EPA and DHA, confirming that milk is an optimal matrix for the provision of important micronutrients.

\section{Acknowledgments}

The study was sponsored by Parmalat S.p.A., Collechio, Parma, Italy (Ivana Gandolfi, PhD and Patrizio Cagnasso, $\mathrm{PhD}$ ), which had no role in study design; in the collection, analysis and interpretation of data; in the writing of the report; and in the decision to submit the article for publication. The authors thank all study participants for their time and enthusiasm. The authors are indebted to Stefania Del Rosso, MD (Ospedale San Raffaele, Milano) for coordinating all clinical laboratory testing, Giorgio Antopulos (Ospedale San Raffaele, Milano) for excellent data management and Lucilla Monti, MD (Ospedale San Raffaele, Milano) for measuring homocysteine for this study. 


\section{References}

[1] The Global Burden of Metabolic Risk Factors for Chronic Diseases Collaboration. Cardiovascular disease, chronic kidney disease, and diabetes mortality burden of cardiometabolic risk factors from 1980 to 2010: a comparative risk assessment. Lancet Diabetes Endocrinol 2014. http://dx.doi.org/10.1016/ S2213-8587(14) 70102-0 [Epub ahead of print].

[2] Messori A, Fadda V, Maratea D, Trippoli S. Omega-3 fatty acid supplements for secondary prevention of cardiovascular disease: from "no proof of effectiveness" to "proof of no effectiveness". JAMA Intern Med 2013;173:1466-8.

[3] Leung Yinko SS, Stark KD, Thanassoulis G, Pilote L. Fish consumption and acute coronary syndrome: a meta-analysis. Am J Med 2014. http://dx.doi.org/ 10.1016/j.amjmed2014.04.016 [Epub ahead of print].

[4] Sinclair AJ, Attar-Bashi NM, Li D. What is the role of alpha-linolenic acid for mammals? Lipids 2002;37:1113-20.

[5] Nguemeni C, Gouix E, Bourourou M, Heurteaux C, Blondeau N. Alpha-linolenic acid: a promising nutraceutical for the prevention of stroke. PharmaNutrition 2013;1:1-8.

[6] Brenna JT, Salem Jr N, Sinclair AJ, Cunnane SC. Alpha-linolenic acid supplementation and conversion to n-3 long-chain polyunsaturated fatty acids in humans. Prostaglandins Leukot Essent Fatty Acids 2009;80:85-91.

[7] Rogers LK, Valentine CJ, Keim SA. DHA supplementation: current implications in pregnancy and childhood. Pharmacol Res 2013;70:13-9.

[8] Visioli F, Rise P, Plasmati E, Pazzucconi F, Sirtori CR, Galli C. Very low intakes of n-3 fatty acids incorporated into bovine milk reduce plasma triacylglycerol and increase HDL-cholesterol concentrations in healthy subjects. Pharmacol Res 2000;41:571-6.

[9] Visioli F, Strata A. Milk dairy products, and their functional effects in humans: a narrative review of recent evidence. Adv Nutr 2014;5:131-43.

[10] Marangoni F, Colombo C, Galli C. A method for the direct evaluation of the fatty acid status in a drop of blood from a fingertip in humans: applicability to nutritional and epidemiological studies. Anal Biochem 2004;326: 267-72.

[11] Bogani P, Galli C, Villa M, Visioli F. Postprandial anti-inflammatory and antioxidant effects of extra virgin olive oil. Atherosclerosis 2007;190:181-6

12] Friedewald WT, Levy RI, Fredrickson DS. Estimation of the concentration of low-density lipoprotein cholesterol in plasma, without use of the preparative ultracentrifuge. Clin Chem 1972;18:499-502.

[13] Heyting A, Tolboom JT, Essers JG. Statistical handling of drop-outs in longitudinal clinical trials. Stat Med 1992:11:2043-61.

[14] Marangoni F, Colombo C, Martiello A, Negri E, Galli C. The fatty acid profiles in a drop of blood from a fingertip correlate with physiological, dietary and lifestyle parameters in volunteers. Prostaglandins Leukot Essent Fatty Acids 2007;76:87-92

[15] Baro L, Fonolla J, Pena JL, Martinez-Ferez A, Lucena A, Jimenez J, Boza JJ, LopezHuertas E. N-3 fatty acids plus oleic acid and vitamin supplemented milk consumption reduces total and LDL cholesterol, homocysteine and levels of endothelial adhesion molecules in healthy humans. Clin Nutr 2003;22:175-82.

[16] Carrero JJ, Baro L, Fonolla J, Gonzalez-Santiago M, Martinez-Ferez A, Castillo R, Jimenez J, Boza JJ, Lopez-Huertas E. Cardiovascular effects of milk enriched with omega-3 polyunsaturated fatty acids, oleic acid, folic acid, and vitamins $\mathrm{E}$ and B6 in volunteers with mild hyperlipidemia. Nutrition 2004;20:521-7.

[17] Benito P, Caballero J, Moreno J, Gutierrez-Alcantara C, Munoz C, Rojo G, Garcia S, Soriguer FC. Effects of milk enriched with omega-3 fatty acid, oleic acid and folic acid in patients with metabolic syndrome. Clin Nutr 2006;25:581-7.

[18] Fonolla J, Lopez-Huertas E, Machado FJ, Molina D, Alvarez I, Marmol E, Navas M, Palacin E, Garcia-Valls MJ, Remon B, Boza JJ, Marti JL. Milk enriched with "healthy fatty acids" improves cardiovascular risk markers and nutritional status in human volunteers. Nutrition 2009;25:408-14.

[19] Debreceni B, Debreceni L. Why do homocysteine-lowering B vitamin and antioxidant e vitamin supplementations appear to be ineffective in the prevention of cardiovascular diseases? Cardiovasc Ther 2012;30:227-33. 